\title{
Analysis of Daily Enhanced Syndromic Surveillance in Hillsborough County, FL, 2015
}

\author{
Charles R. Clark ${ }^{\star 2}$ and Michael Wiese ${ }^{1}$ \\ ${ }^{1}$ Florida Department of Health, Tampa, FL, USA; ${ }^{2}$ Indiana State Department of Health, Indianapolis, IN, USA
}

\section{Objective}

Enhanced daily surveillance is used to identify reportable diseases, outbreaks, and clusters and provides situational awareness. This project examines how health care visits requiring additional information are detected using enhanced syndromic surveillance and the resources required from detection through completion.

\section{Introduction}

The Florida Department of Health in Hillsborough County (FDOHHillsborough) conducts enhanced syndromic surveillance on a daily basis. The Electronic Surveillance System for the Early Notification of Community-based Epidemics in Florida (ESSENCE-FL) is the syndromic surveillance system used by epidemiologists within the Florida Department of Health (FDOH). During the time of this study, ESSENCE-FL receives data from 210 of emergency departments (ED) and 33 urgent care centers (UCC) throughout the state of Florida, including 12 EDs and 3 UCCs in Hillsborough County. In 2014, the ESSENCE-FL system added a feature that delivers an automatic daily email to designated primary ESSENCE-FL users in each county containing all visits which have been detected by the state's visits of interest (VOI) query. The email contains all visits which have been detected by the visits of interest (VOI) query for each ESSENCE-FL users designated county. The VOI query utilizes the combined chief complaint and discharge diagnosis (CCDD) field of a visit for keywords related to reportable diseases and exposures of public health interest. In addition to this VOI email, Hillsborough County analyzes time of arrival alerts, specialized emerging infectious disease queries, poison information center data, and volume levels of syndromes and subsyndromes predetermined by ESSENCE-FL. A daily summary report of the enhanced daily surveillance analysis is then provided to area public health officials within FDOH-Hillsborough and the surrounding counties. This study examines how visits requiring additional investigation are detected and the resources required to complete the investigation.

\section{Methods}

During the study period from July 23 through September 30, 2015, visits identified were recorded along with the time and method of detection. Each day this surveillance began with the review of the visits of interest email, facility and syndrome volumes, the VOI query, emerging infectious disease queries (MERS-CoV, Ebola virus disease, chikungunya, etc.), time of arrival alerts, and the review of Florida Poison Information Center data. A daily summary report of the enhanced surveillance was manually created and provided by email to public health officials. After completion of the daily analysis, facilities were contacted about any visits identified as requiring additional investigation, such as a reportable disease or cluster of public health concern. The time of the information request, receipt of the requested information, and completion of the investigation was recorded.

\section{Results}

An average of 1740 visits were made each day in Hillsborough County in the month prior to the start of this project. During this same time period the daily VOI email identified an average of 5.5 visits per day. During the study period, an average of 7.8 visits were detected each day during the enhanced syndromic surveillance protocol. The VOI email detected 6 visits per day. Overall 558 total visits were detected from the enhanced daily surveillance and 82 percent of these visits were found in the system generated VOI email. Of the visits identified 149 required additional investigation and 15 were determined to be associated with a reportable disease, most commonly carbon monoxide poisoning and varicella. An average of 1.3 days elapsed from the time a visit occurred to the time it was detected through surveillance. Follow-up was started within 1 day of detection and completed in an average of 1.1 days. Overall the daily enhanced syndromic surveillance data analysis required an average of 60 minutes of work time daily with a range of 18-144 minutes.

\section{Conclusions}

During the study period, 15 visits were found to be cases of reportable diseases, primarily carbon monoxide poisoning and varicella, which would have otherwise gone unreported to FDOHHillsborough. The enhanced surveillance process also allows for the quick detection and evaluation of diseases or conditions requiring immediate action that may not always be reported immediately such as meningitis or an emerging infectious disease. The enhanced daily syndromic surveillance in Hillsborough County has been useful in detecting reportable diseases, clusters, and providing situational awareness in a timely manner without an overwhelming burden on staff and resources.

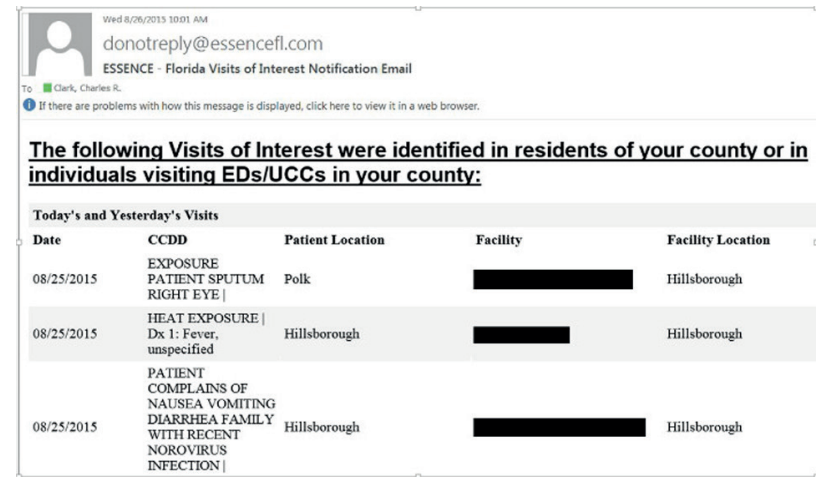

Keywords

ESSENCE; syndromic surveillance; county health department; evaluation; reportable disease

\section{Acknowledgments}

Thanks to Warren McDougle, MPH, David Atrubin, MPH and the entire FDOH-Hillsborough Epidemiology Program for their help and support of this research.

\section{${ }^{*}$ Charles R. Clark}

E-mail: crclark@mac.com 